\title{
Modelo matemático a travez de fórmulas químicas de la viscosidad en soluciones técnicas y puras durante el procesamiento del azúcar de remolacha
}

\begin{abstract}
Mathematical model through chemical formulas of viscosity in technical and pure solutions during processing of beet sugar
\end{abstract}

Javier Ignacio Briones García. ${ }^{1}$, Keren Esther Vásquez Martínez. ${ }^{2}$, Diana Nereida Villa Uvidia. ${ }^{3} \&$ Teresita Jackelin Mejía Reinoso. ${ }^{4}$

Recibido: 10-02-2019 / Revisado: 15-02-209 /Aceptado: 04-03-2019/ Publicado: 14-06-2019

\begin{abstract}
.
DOI: https://doi.org/10.33262/cienciadigital.v3i3.1.704

This research has as a main objective to study the variation of viscosity in the technical and pure solutions in the different stages of the process of sugar using a mathematical model. The optimization of the technological systems in the process of ebullition and crystallization of sugar will improve significantly the technical and economic profit of the plant, in order to carry out this task, it is necessary to control and determine a series of parameters such as: viscosity, saturation, supersaturation, purity of the intercrystalline solution and the molasses. It was carried out through the VisSim 7.0 program, which permitted the modulation of the isobaric evaporative crystallization process to obtain 60 tons of sugar, under the equations of S.V. Shterman, the values of the total duration of the crystallization do not observe pronounced divergence, even though there is a discrepancy for the values of viscosity, which cannot be more than 1500 pascal, for the correct functioning of the vacuum apparatus and to avoid damage. By developing new and revolutionary technologies will permitid to obtain the normal

Escuela Superior Politécnica de Chimborazo, Sede Morona Santiago. Macas, Ecuador. javier.briones@espoch.edu.ec

2 Unidad Educativa Particular Emanuel. Macas, Ecuador. kvasquez@emanuel.edu.ec

3 Escuela Superior Politécnica de Chimborazo, Sede Morona Santiago. Macas, Ecuador. diana.villa@espoch.edu.ec

4 Escuela Superior Politécnica de Chimborazo, Sede Morona Santiago. Macas, Ecuador. teresita.mejia@espoch.edu.ec
\end{abstract}


purity of the intercrystalline solution in the vacuum devices and exclude of the technological line the polythermic crystallization process of saccharose, reducing the costs of operating and expenses in electricity, the use of cooling water consumption and simplify in a great quantity the technology to obtaining sugar.

Keywords: sugar, computer simulation, mathematical model, optimization.

\section{Resumen.}

Esta investigación tiene como objetivo estudiar la variación de la viscosidad en soluciones técnicas y puras en las diversas etapas del procesamiento del azúcar utilizando un modelo matemático. La optimización del régimen tecnológico del proceso de ebullición y cristalización del azúcar mejorará significativamente el rendimiento técnico y económico de la planta, para realizar dicha tarea, es necesario controlar y determinar una serie de parámetros como: viscosidad, saturación, sobresaturación, pureza de la solución intercristalina y melaza. Se llevó a cabo mediante el programa VisSim 7.0, el cual permitió la modulación del proceso de cristalización evaporativa isobárica para la obtención de 60 toneladas azúcar, bajo las ecuaciones de S.V. Shterman, los valores de la duración total de la cristalización no observan divergencia pronunciada, aunque existe discrepancia para los valores de viscosidad, los cuales no deben ser mayor a 1500 pascal, para el correcto funcionamiento del aparato de vacío y evitar daños. Al desarrollar nuevas y revolucionarias tecnologías permitirán obtener la pureza normal de la solución intercristalina en los aparatos de vacío y excluir de la línea tecnológica el proceso de cristalización politérmica de la sacarosa, reduciendo costos de operación, gastos en electricidad, consumo de agua refrigerante y simplificaría en gran medida la tecnología de obtención de azúcar.

Palabras claves: azúcar, simulación computarizada, modelo matemático, optimización.

\section{Introducción.}

La remolacha azucarera es la principal fuente de obtención de azúcar en muchos países, siendo el $90 \%$ de azúcar consumida en Europa a base de remolacha azucarera y el $30 \%$ a nivel mundial, gracias a su elevado contenido de sacarosa. En la producción de este tipo de azúcar, intervienen procesos físicos y químicos complejos, donde la sacarosa es extraída de las células en difusión por acción química y termo-física separando los azúcares de los noazúcares convirtiéndolo en un producto cristalino puro. 
Cocción del jarabe - Es la etapa final en la producción del azúcar. El objetivo principal es la extracción de la sacarosa disuelta en el almíbar, en forma de cristales. Durante el proceso de purificación llamado carbonatación - el líquido resultante del proceso de difusión se mezcla con lechada de cal $\left[\mathrm{Ca}(\mathrm{OH}) \_2\right]$ y gas carbónico ( $\llbracket \mathrm{CO} \rrbracket \_2$ ), produciendo carbonato de calcio ( $\llbracket \mathrm{CaCO} \rrbracket \_3$ ) que se precipita, arrastrando la mayor parte de las impurezas consigo, en el proceso se elimina un tercio de los no azucares.

Las sustancias restantes junto con la sacarosa son redirigidas al departamento de producción, donde el jarabe pasa por el proceso de evaporación hasta que llegue a una concentración de 70-75\% de contenido sólidos, después de lo cual está listo para su cristalización. El azúcar cristalino se obtiene mediante dos técnicas:

- Cristalización politérmica;

- Cristalización por evaporación isobárica.

La separación de la sacarosa es realizada en dos o tres etapas, teniendo en cuenta la calidad de la materia prima. En el caso de materias primas de baja calidad, el azúcar es obtenida mediante dos esquemas de cristalización (cristalización politérmica). Por lo contrario, si la materia prima es de alta calidad se utilizan tres esquemas (cristalización por evaporación isobárica).

Los investigadores centran cada vez más su atención en el desarrollo de nuevas tecnologías de cristalización, debido al gran contenido de impurezas acumuladas en la solución madre, trayendo como resultado, mayor viscosidad y dificultad en el proceso de cristalización, disminuyendo el volumen final del producto. La reducción de la pureza de la melaza ha generado un mayor rendimiento productivo, pero ha alcanzado límites prácticos, llevando resultados indeseables.

Esto se debe a la elevada viscosidad de la solución intercristalina, lo que empeora las condiciones de nucleación, crecimiento cristalino y duración del proceso debido a una disminución en la velocidad de cristalización. Dado que la pureza de la melaza depende de la calidad de los productos que entran en ebullición, como del retorno y recirculación del licor madre, esto conduce a la gran acumulación de no-azucares, lo que afecta negativamente al rendimiento técnico y económico de la planta.

La modelación matemática en los últimos años ha sido un método efectivo y comúnmente utilizado en el desarrollo y estudio de procesos tecnológicos, proporcionando respuestas a muchas preguntas incluso en la etapa preliminar de investigación, permitiendo determinar modos óptimos y esquemas apropiados en los procesos tecnológicos. Eliminando los gastos innecesarios de mano de obra y recursos materiales en la construcción de esquemas irracionales y en la implementación de regímenes ineficientes. El modelo matemático es una herramienta extremadamente flexible que permite reproducir cualquier situación, tanto real como hipotética, ya que no está sujeta a limitaciones físicas. Esto reduce la necesidad de 
sofisticados equipos de laboratorio y pruebas operacionales de los procesos tecnológicos que se están creando.

\section{Metodologia.}

El análisis del material bibliográfico permitió concluir, que la viscosidad depende ampliamente de la densidad, temperatura, composición química y asociación de las moléculas en las distintas soluciones. Cuanto más complicada sea la separación de la solución intercristalina de los cristales de sacarosa, mayor será la concentración de noazúcares en el licor madre, requiriendo una mayor cantidad de equipos para su procesamiento.

Numerosos estudios han demostrado que la viscosidad de las soluciones técnicas de azúcar, incluyendo la melaza, no dependen de la velocidad de cizallamiento, es decir, estos fluidos son newtonianos. Sin embargo, estudios más detallados han demostrado el carácter no newtoniano de algunas melazas y que las propiedades de las soluciones de azúcar son similares a las soluciones de polímeros y de sistemas dispersos.

Estas desviaciones se deben a la presencia de burbujas de gas, altos polímeros y sólidos suspendidos en las soluciones. Que en comparación con soluciones puras de sacarosa, que contienen no-azucares, durante una misma concentración de sólidos tiene una viscosidad más baja. Pero esto sólo aplicaría a soluciones que no contengan pectinas, dextranos, polímeros cuya presencia aumenta considerablemente la viscosidad.

Diferentes profesionales de la industria azucarera han basado sus trabajos investigativos en el estudio de la viscosidad en las diferentes etapas que presentan las soluciones, para la obtención de los cristales de azúcar, dichas investigaciones han podido generar ecuaciones que describen la dependencia de esta, desde diferentes variables formando un grupo de 17 ecuaciones, las cuales se muestran en la tabla 1. Sin embargo es imposible compararlas entre sí, ya que la literatura no cuenta con valores de constantes para cada ecuación.

Tabla 1: Ecuaciones de viscosidad de soluciones técnicas y puras de la sacarosa

\begin{tabular}{|l|l|c|}
\hline$№$ & AUTORES & ECUACIÓN DE VISCOSIDAD \\
\hline 1 & G. Waterman & $\lg \mu=\frac{A}{T x+B}$ \\
\hline 2 & J. Pidu & $\lg \mu=\frac{A+B\left(T-T_{0}\right)}{T^{2}}$ \\
\hline 3 & Y. Frankel & $\mu=A e^{E v / R T}$ \\
\hline 4 & $\begin{array}{l}\text { R. Mishchuk, L. } \\
\text { Belostotsky }\end{array}$ & $E=2.303 R * \frac{d(\lg \mu)}{d(1 / T)}$ \\
\hline 5 & G. Znamenskii & $\mu=\mu_{B} * A^{x}, \mu=\mu_{p}(3.5-0.025 * P)$ \\
\hline 6 & D. Shliphake & $\mu_{M}=\mu_{100} *\left[1-\frac{1-P_{M}}{0.4} *\left(4.734-0.0379 * t_{y}\right) * \frac{C B}{1900-18 * C B_{M}}\right.$ \\
\hline
\end{tabular}




\begin{tabular}{|c|c|c|}
\hline 7 & K. Wagner & $\lg \mu_{120}=\frac{C B}{(1900-18 * C B)} *\left(\frac{4120}{t+91}-11.75\right)-3.1$ \\
\hline 8 & J. Jhonatelle & $\lg \mu=22.46 * N-2.114+\frac{30-t}{91-t} *\left(1.1+43.1 * A * N^{1.25}\right)$ \\
\hline 9 & N. Lyusy & $\mu_{y}=\frac{\mu_{M P}}{\left(1-0.03569 * K+0.00322 * K^{2}\right)}$ \\
\hline 10 & V. Shterman & $\lg \mu_{M}=-0.19 *\left[32.89-\frac{3.131 * 10^{4} * t}{(t+273.15)^{2}}\right] *\left(N_{C X}+K_{\mu} * N_{H C}\right)$ \\
\hline 11 & $\begin{array}{l}\text { V. Shterman, A. } \\
\text { Filippov }\end{array}$ & $\lg \mu=-0.1096+\left[37.66-129.3\left(T_{0}{ }_{T}\right)+124.78\left(T_{0} T_{T}\right)^{2}\right] N_{S}=a_{0}+a_{1}(t) N_{S}$ \\
\hline 12 & S. Petrov & $\begin{array}{c}\lg \mu=\frac{0,00292 \cdot 10^{-4} \cdot \mathrm{CB} \cdot \mathrm{Y}+\mathrm{a}_{1} \cdot 10^{-4}(100-\mathrm{Y}) \cdot \mathrm{CB}+\mathrm{a}_{2} \cdot 0,05556 \cdot 10^{-2}(100-\mathrm{CB})}{0,00292 \cdot 10^{-4} \cdot \mathrm{CB} \cdot \mathrm{\Psi}+0,05556 \cdot 10^{-2} \cdot(100-\mathrm{CB})+\mathrm{a}_{3} \cdot 10^{-4}(100-\mathrm{Y}) \cdot \mathrm{CB}} \\
+\left(a_{4}+\frac{a_{5} * t}{\left(a_{6}+t\right)^{2}}\right)+a_{7}\end{array}$ \\
\hline 13 & B. Maurandi & $\mu_{M}=\mu_{M 0} *\left[0.525 * 100-P_{M} / P_{M}\right]^{10 \cdot(P a-1)}$ \\
\hline 14 & V. Shterman, G. Vovk & $\lg \mu=a_{0}+a_{1}(t)\left[p+k(1-p) N_{d}=a_{0}+a_{1}(t) q N_{d}\right.$ \\
\hline 15 & V. Shterman & $m u y=\frac{m u m}{\left[1-3.4 * g a m y * d k p^{(-0.0525)}+3.01 * g a m y^{2} * d k p^{(-0.0965)}\right]}$ \\
\hline 16 & N. Silina & $\mu_{0}=\frac{\mu_{y}}{\mu}=\frac{1}{1-3,8047 F+3,6584 F^{2}}$ \\
\hline 17 & $\begin{array}{l}\text { A. Gromkovsky, V. } \\
\text { Fursov }\end{array}$ & $\frac{\mu_{y}}{\mu}=\frac{1}{1-3,4 \varphi * d^{-0,0525}+3,01 \varphi * d^{-0,0965}}$ \\
\hline
\end{tabular}

Elaborado: Por los autores

En base a los cambios en la viscosidad de soluciones técnicas y puras de la sacarosa en el proceso de ebullición y cristalización se tomo como referencia las ecuaciones creadas por Sergey Shterman las cuales coinciden con la línea investigativa del proceso planteado.

Primera ecuación de S. Shterman

$$
\begin{aligned}
& \text { mum }=a 0+a 1 \cdot[D B m 3+k \cdot(1-D B m 3)] \cdot N c v 3 \\
& a 0=-0.10906 \\
& a 1=37,66-129,3 \cdot\left[\frac{273}{(T y 3+273)}\right]+124,78 \cdot\left[\frac{273}{(T y 3+273)}\right] \\
& N c v 3=\frac{C V m 3}{[C V m 3=19 \cdot(1-C V m 3)]} \\
& m u y=\frac{\mathrm{mum}}{\left[1-3.4 \cdot g a m y \cdot d k r^{-0.0525}+3.01 \cdot \mathrm{gamy}^{2} \cdot \mathrm{dkr}-0.0965\right]}
\end{aligned}
$$

En donde:

mum = viscosidad de la solución intercristalina; $\mathrm{DBm} 3=$ pureza de la solución intercristalina; $\mathrm{CVm} 3$ = sólidos en la solución intercristalina; $\mathrm{K}=$ coeficiente de impurezas sobre la viscosidad de soluciones técnicas azucaradas; Ncv3 = fracción molar de sólidos en la solución intercristalina; muy = viscosidad de la masa cocida; gamy = fracción de volumen de los cristales en la masa cocida; $\mathrm{dkr}=$ tamaño de los cristales. 
Viscosidad por la segunda ecuación de S. Shterman:

$$
\begin{aligned}
& \text { mum } 1=\left\{\exp \left[2.3026 \cdot\left(\frac{2.89-31310 \cdot T y 3}{(T y 3+273.15)^{2} \cdot(N c x 3+k \cdot N n c x 3)-0.19}\right)\right]\right\} \\
& N c v 3=\frac{C V m 3}{[C V m 3+19 \cdot(100-C V m 3)]} \\
& N n c x 3=\frac{(C V m 3-0.01 \cdot D B m 3 \cdot C V m 3)}{[C V m 3+19 \cdot(100-C V m 3)]}
\end{aligned}
$$

Donde:

mum1 = viscosidad de la solución intercristalina; Ty3 - temperatura de la masa de cocion; Ncv3 y Nncx3 = fracción molar de las sustancias solidas y los no azucares.

Por consiguiente, la viscosidad se describe mediante la ecuación:

$$
m u y 1=\frac{m u m 1}{\left(1-3.4 \cdot g a m y \cdot d k r^{-0.0525}+3.01 \cdot g a m y^{2} \cdot d k r^{-0.0965}\right)}
$$

La investigación se implementó eficazmente utilizando el modelo matemático (VisSim 7.0), VisSim - es un lenguaje visual de programación, diseñado para modelar sistemas dinámicos, así como proyecciones basadas en modelos matemáticos.

VisSim combina un ensamblado de la interfaz intuitiva de arrastrar y colocar los diagramas de bloques a un motor de simulación de gran alcance. El lenguaje de programacion fue desarrollado por la compañía americana Visual Solutions, que se encuentra en Westford (Massachusetts).

La ventaja de este modelo es que con su ayuda es posible demostrar, desarrollar y analizar efectivamente nuevas tecnologías de ebullición y cristalización de masas cocidas, alcanzando 60 toneladas de producto final y la concentracion requerida en la masa cocida, independientemente de los parametros tecnologicos, fisico-quimicos y termo-tecnicos del proceso.

Está diseñado para estudiar, fundamentar nuevas tecnologías de cristalización sobre la base del estudio y procesamiento de los resultados de un experimento informático durante el modelado matemático del proceso de cristalización industrial, por ejemplo, la sacarosa

La simulacion se llevo a cabo tomando en cuentas los siguientes parametros tecnologicos:

- Modo típico de ebullición, dentro del cual se mantuvo una sobresaturación constante de $\mathrm{P}=$ const y el final del proceso fue determinado por la materia seca de la masa cocida. En este caso, la cantidad final de la masa cocida es siempre 
diferente, dependiendo de los parámetros tecnológicos y de la composición de las impurezas.

- Modo de soldadura con control automático. En este caso, la cantidad final de la masa cocida y las sustancias secas permanecen inalteradas, y en cualquier caso, hasta el final del proceso de ebullición, los valores de volumen de producto final alcanzando 60 toneladas, con un coeficiente de sobresaturación dado.

- El modo de cristalización cuando se alcanza la frecuencia normal, que es la base del modo típico.

- El regimen de cristalización cuando se alcanza la pureza mínima.

- El régimen de cristalización con la selección de cristales a sobresaturación constante es $\mathrm{P}=$ const.

\section{Resultados.}

La comparación de las ecuaciones de Sergey Shterman se llevó a cabo utilizando el modelo matemático antes mencionado, dando como resultado que las primeras ecuaciones descritas por el autor ruso corresponden a datos referenciales de parámetros finales para la melaza, pero la viscosidad es mucho mayor que en las ecuaciones mas recientemente creadas por este, afirmando que estas poseen datos mucho mas precisos (Shterman, 2006).

S.V. Shterman enfatiza tomar en cuenta el efecto de los no-azúcares sobre las principales características tecnológicas de los productos de ebullición y cristalización con la ayuda de coeficientes $\mathrm{m}$, b y k. Los dos primeros coeficientes reflejan la influencia de los no azucares sobre la solubilidad de la sacarosa, y la segunda sobre la viscosidad de las soluciones técnicas de azúcar.

Figura 1: Comparación de las ecuaciones 1, 4, 5, 8

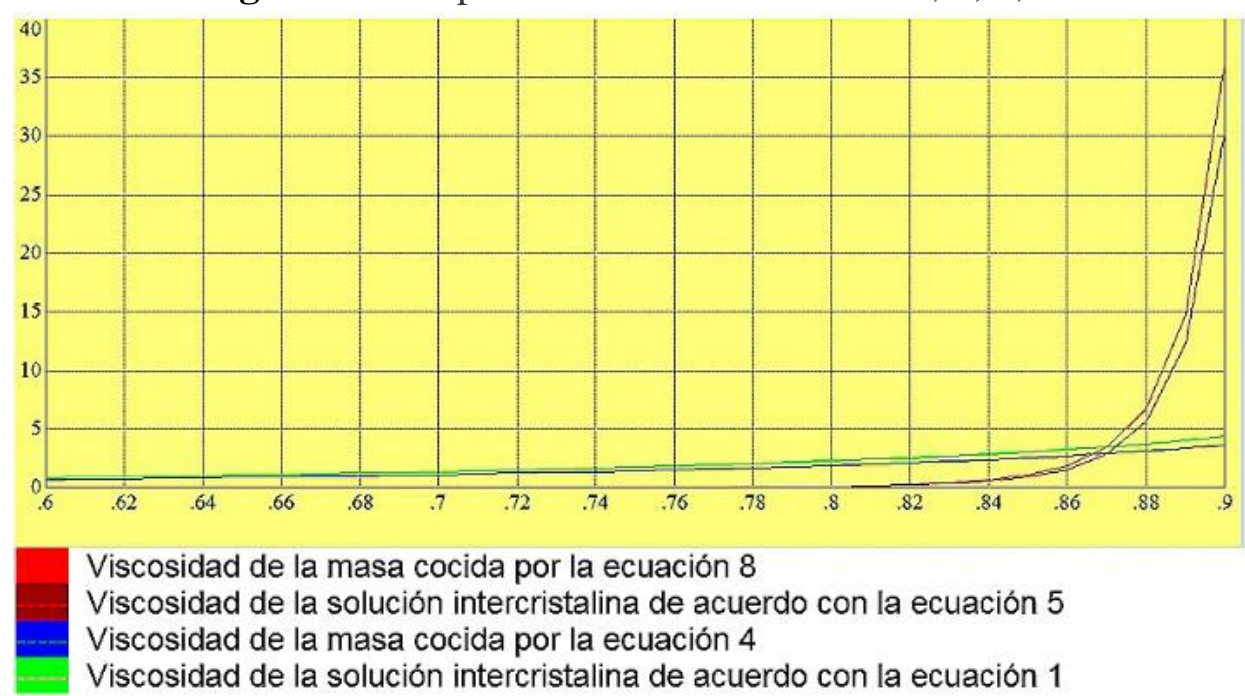

Fuente: Los autores 
Con el programa informático, se llevó a cabo una serie de experimentos, para estudiar el proceso de ebullición del licor madre por las ecuaciones de viscosidad de Shterman, para el ultimo producto con los siguientes datos:

Concentración de sólidos - 0,94 / 0,95 / 0,96, pureza - 0,76. El coeficiente "m" varía en el intervalo de 0,1 a 0,$5 ; \mathrm{B}=1-\mathrm{m}$, los coeficientes "c" y "k" se asumieron sin cambios, los datos se resumen en las tablas 2, 3 y 4 . El programa proporcionó las siguientes funciones:

El modelado y control del proceso de cristalización por evaporación isobárica en la zona de crecimiento cristalino metaestable manteniendo la relación calculada de la masa de agua evaporada y el producto cristalizable a añadir. Al mismo tiempo, el control del proceso se llevó a cabo de tal manera que el producto cristalizado, como regla, necesariamente alcanza la concentración especificada y el volumen requerido del producto cristalizado, dependiendo del tipo y diseño del equipo utilizado.

Además, el programa permitió explorar a fondo la cinética y la dinámica del proceso de cristalización, estudiar los patrones de cambios en la tecnología, la ingeniería térmica, los indicadores físico-químicos y otros del proceso, sobre esta base justificar y desarrollar nuevas tecnologías para la cristalización de la sacarosa.

Figura 2: Modelo matemático por las ecuaciones de S. V. Shterman 


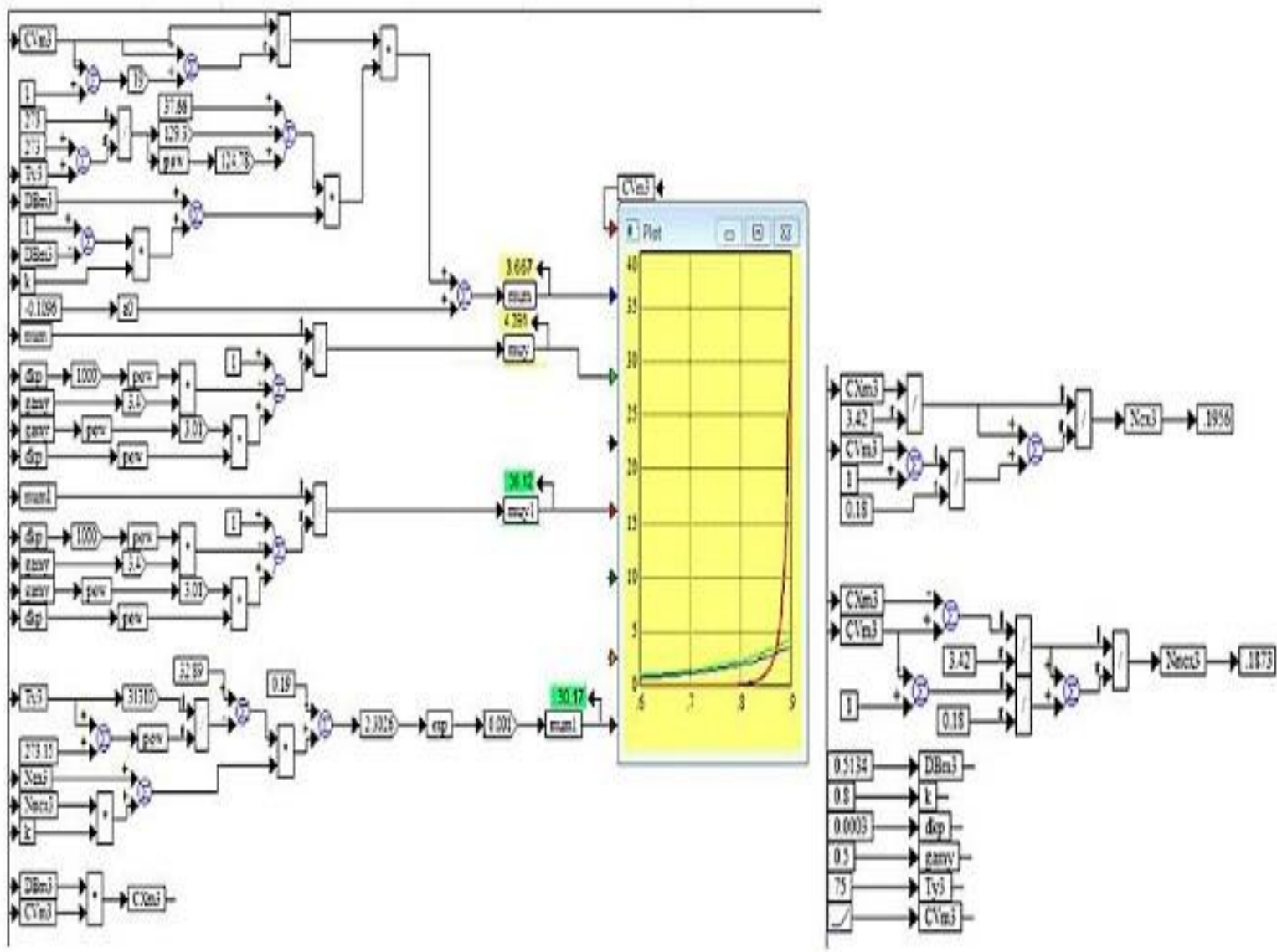

Fuente: Los autores

En las tablas 2, 3 y 4 se muestra como resultado que el menor tiempo de cristalización proporciona la ecuación 2 con parámetro $\mathrm{b}=0.5 \mathrm{y} \mathrm{m}=0.5$, el análisis de las mediciones en la viscosidad de la masa cocida y de la solución intercristalina durante el proceso de ebullición y cristalización con el uso del modelo matemático ha demostrado que la regularidad de la medicion en la viscosidad con la composición de impurezas depende fuertemente de los valores integrales de los coeficientes $\mathrm{m}, \mathrm{b}, \mathrm{k}$, los dos primeros coeficientes reflejan la influencia de los no azucares sobre la solubilidad de la sacarosa, y la segunda sobre la viscosidad de las soluciones técnicas de azúcar. 
Tabla 2: Masa cocida de producto final con parámetros: Solidos $=0,94 ;$ pureza $=0,76 ; b=0.9 ; 0.7 ; 0.5$

\begin{tabular}{|c|c|c|c|c|c|c|c|c|c|}
\hline \multirow{2}{*}{$\begin{array}{l}\text { Parámetros de } \\
\text { investigación }\end{array}$} & \multicolumn{2}{|c|}{$\mathrm{m}=0.1$} & \multirow{2}{*}{$\begin{array}{c}\text { Cambios en } \\
\% \\
\end{array}$} & \multicolumn{2}{|c|}{$\mathrm{m}=0.3$} & \multirow{2}{*}{$\begin{array}{c}\text { Cambios } \\
\text { en } \%\end{array}$} & \multicolumn{2}{|c|}{$\mathrm{m}=0.5$} & \multirow{2}{*}{$\begin{array}{c}\text { Cambios en } \\
\%\end{array}$} \\
\hline & Ecuación 1 & Ecuación 2 & & Ecuación 1 & Ecuación 2 & & Ecuación 1 & Ecuación 2 & \\
\hline $\begin{array}{l}\text { Tiempo total de } \\
\text { cristalización } \mathbf{t}\end{array}$ & 7.82 & 7.9 & $+1 \%$ & 5.75 & 5.65 & $-1.7 \%$ & 5.25 & 4.51 & $-14 \%$ \\
\hline $\begin{array}{l}\text { Masa de jarabe añadido } \\
\text { mcd3 }\end{array}$ & 67692.2 & 67692.3 & $0 \%$ & 66769.6 & 66766.7 & $-0.004 \%$ & 66769.2 & 66774.6 & $+0.008 \%$ \\
\hline $\begin{array}{l}\text { Masa general de agua } \\
\text { evaporada MviOb }\end{array}$ & 27694 & 27694.4 & $0 \%$ & 26771.5 & 26772.7 & $+0.004 \%$ & 26770 & 26772.9 & $+0.01 \%$ \\
\hline $\begin{array}{l}\text { Cantidad total de azúcar } \\
\text { cristalizada Mkrob3 }\end{array}$ & 26396.9 & 26403.7 & $+0.02 \%$ & 16335.9 & 16345.4 & $+0.5 \%$ & 8827.2 & 8834.12 & $+0.07 \%$ \\
\hline $\begin{array}{l}\text { Viscosidad de la masa } \\
\text { cocida muy }\end{array}$ & 325.5 & 5.56 & $-98.3 \%$ & 330.2 & 8.124 & $-97.4 \%$ & 1068 & 7.642 & $-99.6 \%$ \\
\hline $\begin{array}{l}\text { Concentración final de } \\
\text { solución intercristalina } \\
\text { Cvm3 }\end{array}$ & 0.910758 & 0.910763 & $+0.005 \%$ & 0.91759 & 0.917624 & $+0.002 \%$ & 0.92966 & 0.929678 & $+0.001 \%$ \\
\hline $\begin{array}{l}\text { Pureza final de la } \\
\text { solución intercristalina } \\
\text { Dbm3 }\end{array}$ & 0.552993 & 0.552895 & $-0.02 \%$ & 0.66214 & 0.662061 & $-0.01 \%$ & 0.71547 & 0.715433 & $-0.005 \%$ \\
\hline $\begin{array}{l}\text { Viscosidad de la } \\
\text { solución intercristalina } \\
\text { mum }\end{array}$ & 25.14 & 3.561 & $-86.5 \%$ & 95.29 & 4.093 & $-96.2 \%$ & 594 & 4.736 & $-99.8 \%$ \\
\hline $\begin{array}{l}\text { Tamaño final del cristal } \\
\text { dkp }\end{array}$ & 0.000376 & 0.000376 & $0 \%$ & 0.00032 & 0.00032 & $0 \%$ & 0.00026 & 0.000261 & $0 \%$ \\
\hline
\end{tabular}

Fuente: Los autor 
Tabla 3: Masa cocida de producto final con parámetros: Solidos $=0,95 ;$ pureza $=0,76 ; b=0.9 ; 0.7 ; 0.5$

\begin{tabular}{|c|c|c|c|c|c|c|c|c|c|}
\hline \multirow{2}{*}{$\begin{array}{l}\text { Parámetros de } \\
\text { investigación }\end{array}$} & \multicolumn{2}{|c|}{$\mathrm{m}=0.1$} & \multirow{2}{*}{$\begin{array}{c}\text { Cambios en } \\
\% \\
\end{array}$} & \multicolumn{2}{|c|}{$\mathrm{m}=0.3$} & \multirow{2}{*}{$\begin{array}{l}\text { Cambios } \\
\text { en } \%\end{array}$} & \multicolumn{2}{|c|}{$\mathrm{m}=0.5$} & \multirow{2}{*}{$\begin{array}{c}\text { Cambios en } \\
\% \\
\end{array}$} \\
\hline & Ecuación 1 & Ecuación 2 & & Ecuación 1 & Ecuación 2 & & Ecuacion 1 & Ecuacion 2 & \\
\hline $\begin{array}{l}\text { Tiempo total de } \\
\text { cristalización } \mathbf{t}\end{array}$ & 7.82 & 7.9 & $+1 \%$ & 6.32 & 6.09 & $-3.6 \%$ & 6.35 & 4.92 & $-22 \%$ \\
\hline $\begin{array}{l}\text { Masa de jarabe } \\
\text { añadido mcd3 }\end{array}$ & 67692.2 & 67692.3 & $0 \%$ & 67692.1 & 67689.5 & $-0.003 \%$ & 67692.3 & 67696.3 & $+0.005 \%$ \\
\hline $\begin{array}{l}\text { Masa general de agua } \\
\text { evaporada MviOb }\end{array}$ & 27694 & 27694.4 & $0 \%$ & 27693.5 & 27697.9 & $+0.01 \%$ & 27694 & 27694 & $0 \%$ \\
\hline $\begin{array}{l}\text { Cantidad total de } \\
\text { azúcar cristalizada } \\
\text { Mkrob3 }\end{array}$ & 26396.9 & 26403.7 & $+0.02 \%$ & 18205 & 18219 & $+0.07 \%$ & 10125.9 & 10131.9 & $+0.05 \%$ \\
\hline $\begin{array}{l}\text { Viscosidad de la masa } \\
\text { cocida muy }\end{array}$ & 325.5 & 5.56 & $-98.3 \%$ & 1514 & 8.835 & $-99.8 \%$ & 6191 & 8.872 & $-99.6 \%$ \\
\hline $\begin{array}{l}\text { Concentración final de } \\
\text { solución } \\
\text { intercristalina Cvm3 }\end{array}$ & 0.910758 & 0.910763 & $+0.005 \%$ & 0.928249 & 0.928341 & $+0.009 \%$ & 0.939866 & 0.939874 & $+0.008 \%$ \\
\hline $\begin{array}{l}\text { Pureza final de la } \\
\text { solución } \\
\text { intercristalinaDbm3 }\end{array}$ & 0.552993 & 0.552895 & $-0.02 \%$ & 0.647381 & 0.647249 & $-0.2 \%$ & 0.708158 & 0.708123 & $-0.004 \%$ \\
\hline $\begin{array}{l}\text { Viscosidad de la } \\
\text { solución } \\
\text { intercristalinamum }\end{array}$ & 25.14 & 3.561 & $-86.5 \%$ & 355.4 & 4.452 & $-98.6 \%$ & 3104 & 5.194 & $-99.3 \%$ \\
\hline $\begin{array}{l}\text { Tamaño final del } \\
\text { cristal dkp }\end{array}$ & 0.000376 & 0.000376 & $0 \%$ & 0.000332 & 0.000332 & $0 \%$ & 0.000273 & 0.000273 & $0 \%$ \\
\hline
\end{tabular}

Fuente: Los autores

Tabla 4: Masa cocida de producto final con parámetros: Solidos $=0,95 ;$ pureza $=0,76 ; b=0.9 ; 0.7 ; 0.5$ 


\begin{tabular}{|c|c|c|c|c|c|c|c|c|c|}
\hline \multirow{2}{*}{$\begin{array}{l}\text { Parámetros de } \\
\text { investigación }\end{array}$} & \multicolumn{2}{|c|}{$\mathrm{m}=0.1$} & \multirow{2}{*}{$\begin{array}{c}\text { Cambios en } \\
\%\end{array}$} & \multicolumn{2}{|c|}{$\mathrm{m}=0.3$} & \multirow{2}{*}{$\begin{array}{l}\text { Cambios } \\
\text { en } \%\end{array}$} & \multicolumn{2}{|c|}{$\mathrm{m}=0.5$} & \multirow{2}{*}{$\begin{array}{c}\text { Cambios en } \\
\%\end{array}$} \\
\hline & Ecuación 1 & Ecuación 2 & & Ecuación 1 & Ecuación 2 & & Ecuacion 1 & Ecuacion 2 & \\
\hline $\begin{array}{l}\text { Tiempo total de } \\
\text { cristalización } \mathbf{t}\end{array}$ & 8.69 & 8.73 & $+0.5 \%$ & 7.1 & 6.57 & $-8 \%$ & 8.55 & 5.38 & $-37.4 \%$ \\
\hline $\begin{array}{l}\text { Masa de jarabe } \\
\text { añadido med3 }\end{array}$ & 68615.4 & 68615.8 & $0 \%$ & 68615.4 & 68615 & $0 \%$ & 68615.4 & 68615.9 & $0 \%$ \\
\hline $\begin{array}{l}\text { Masa general de agua } \\
\text { evaporada MviOb }\end{array}$ & 28617.5 & 28617 & $0 \%$ & 28617 & 28617.5 & $0 \%$ & 28616 & 28616.5 & $0 \%$ \\
\hline $\begin{array}{l}\text { Cantidad total de } \\
\text { azúcar cristalizada } \\
\text { Mkrob3 }\end{array}$ & 28897.7 & 28891.7 & $-0.02 \%$ & 20083.2 & 20086.4 & $+0.01 \%$ & 11422.5 & 11426.5 & $+0.03 \%$ \\
\hline $\begin{array}{l}\text { Viscosidad de la masa } \\
\text { cocida muy }\end{array}$ & 1565 & 5.26 & $-99.3 \%$ & 11631 & 9.515 & $-99.7 \%$ & 62784.5 & 10.36 & $-99.9 \%$ \\
\hline $\begin{array}{l}\text { Concentración final de } \\
\text { solución } \\
\text { intercristalinaCvm3 }\end{array}$ & 0.92289 & 0.92287 & $-0.002 \%$ & 0.93990 & 0.93992 & $+0.002 \%$ & 0.95960 & 0.95060 & $0 \%$ \\
\hline $\begin{array}{l}\text { Pureza final de la } \\
\text { solución } \\
\text { intercristalinaDbm3 }\end{array}$ & 0.51837 & 0.51848 & $+0.02 \%$ & 0.63153 & 0.63149 & $-0.006 \%$ & 0.70063 & 0.70061 & $-0.002 \%$ \\
\hline $\begin{array}{l}\text { Viscosidad de la } \\
\text { solución } \\
\text { intercristalinamum }\end{array}$ & 79.34 & 3.847 & $-95.4 \%$ & 2169 & 4.91 & $-99.8 \%$ & 28195.9 & 5.77 & $-99.9 \%$ \\
\hline $\begin{array}{l}\text { Tamaño final del } \\
\text { cristal dkp }\end{array}$ & 0.000387 & 0.000387 & $0 \%$ & 0.000343 & 0.000343 & $0 \%$ & 0.000284 & 0.000284 & $0 \%$ \\
\hline
\end{tabular}

Fuente: Los Autores 
La simulación de procesos por computadora, ha permitido optimizar las diversas etapas de los diferentes esquemas tecnológicos utilizados por las industrias, evitando gastos innecesarios en la implementación de regímenes y equipos ineficientes. Con el paso del tiempo la industria azucarera ha evolucionado, con la finalidad de aumentar su producción y disminuir perdidas del producto, gracias a la implementación de equipos que aceleran la saturación de la melaza y permiten el control automático de su viscosidad.

La cristalización del azúcar es considerada una de las etapas científicas-técnicas de mayor interés durante el procesamiento de la remolacha, porque allí ocurre la principal perdida de azúcar, alcanzando un $75 \%$ de pérdida total dentro de la producción. La modelación de estos procesos permite determinar los parámetros óptimos para la obtención de una melaza normal sin gastos para la empresa.

En el marco de solución, se proponen y justifican las siguientes variantes tecnologicas:

- Disminución de la viscosidad de la solución intercristalina disminuyendo la sobresaturación, comenzando desde el momento en que se alcanza la viscosidad máxima 50 ... 70 Pa.s.

- $\quad$ Disminución de la viscosidad de la masa cocida debido a la selección de masa cristalina, a partir del momento en que se alcanza la viscosidad máxima 50 ... 70 Pa.s.

- $\quad$ Disminución de la viscosidad de la masa cocida debido a un cambio en la pureza del producto añadido.

- $\quad$ Disminución de la viscosidad de la masa cocida debido a una combinación de variantes de los métodos anteriores.

\section{Conclusiones.}

- El análisis bibliográfico arrojo 17 ecuaciones de diferentes autores referentes a la viscosidad en soluciones técnicas y puras durante el proceso de ebullición y cristalización de la sacarosa.

- Se construye un modelo matemático que proporciona 60 toneladas de producto final con las ecuaciones de S. V. Shterman, tomando en cuenta que las soluciones en el aparato de vacío no pueden superar los 1500 pascal de viscosidad para evitar daños y perdida del producto.

- Los valores de viscosidad según las ecuaciones de Shterman mostraron diferencia, pero los resultados de la modulación del proceso de cristalización evaporativa isobárica en los valores de la duración total de la cristalización no observan divergencia pronunciada.

- La validación de la modelación computacional reduciría significativamente los costos de operación de equipos voluminosos, los gasto en electricidad, el consumo de agua refrigerante y simplificaría en gran medida la tecnología de obtención de azúcar. 


\section{Referencias bibliográficas.}

Sapronov, A. (2014). Tecnologia de la produccion de azucar. Kolos. 150-250.

Sherman, S. (2007). La composición y viscosidad óptimas del procesamiento del azucar de romolacha y de la última cristalización en varias etapas de su producción. Sahar, 5. 160.

Sherman, V. VOVK, G. (2006). Descripción analítica de la viscosidad de las soluciones técnicas de azúcar y melaza.Sahar,3. 250.

Slavyanckiy, A. (2006). Equipos tecnológicos de las fábricas de azúcar: clasificación, características técnicas, cálculos, diseño. MGUPP. 60-100.

Slavyanckiy, A.Goldenberg, S. (2004). Cálculo de productos de un esquema tecnológico típico y el uso de computadoras para mejorar la eficiencia de la producción de azúcar. MGUPP. 98.

Tuzhilkin, V. (2007). Cristalizacion del azucar. MGUPP. 336.

Zaets, A. (2014). Regularizacion del azucar: problemas metodologicos de su creacion. Sahar, 5. $17-20$ 


\section{PARA CITAR EL ARTÍCULO INDEXADO.}

Briones García, J., Vásquez Martínez, K., Villa Uvidia., D., \& Mejía Reinoso, T. (2019). Modelo matemático a travez de fórmulas químicas de la viscosidad en soluciones técnicas y puras durante el procesamiento del azúcar de remolacha. Ciencia Digital, 3(3.1), 295-309. https://doi.org/10.33262/cienciadigital.v3i3.1.704

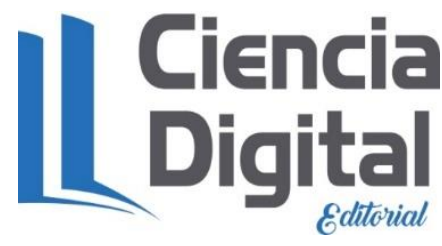

El artículo que se publica es de exclusiva responsabilidad de los autores y no necesariamente reflejan el pensamiento de la Revista Ciencia Digital.

El artículo queda en propiedad de la revista y, por tanto, su publicación parcial y/o total en otro medio tiene que ser autorizado por el director de la Revista Ciencia Digital.
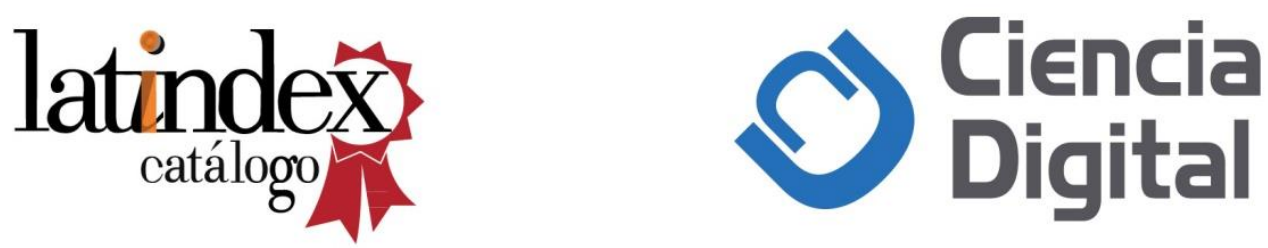